\title{
La coopération décentralisée face aux enjeux du développement durable
}

Introduction au numéro « Coopération décentralisée et développement durable »

Decentralized cooperation and the challenges of sustainable development

\section{Abdelkader Djeflat et Bruno Boidin}

\section{(2) OpenEdition}

\section{Journals}

Édition électronique

URL : http://journals.openedition.org/developpementdurable/8387

DOI : 10.4000/developpementdurable.8387

ISSN : 1772-9971

Éditeur

Association DD\&T

Référence électronique

Abdelkader Djeflat et Bruno Boidin, « La coopération décentralisée face aux enjeux du développement durable», Développement durable et territoires [En ligne], Vol. 1, n 1 | Mai 2010, mis en ligne le 07 mai 2010, consulté le 21 septembre 2020. URL : http://journals.openedition.org/developpementdurable/ 8387 ; DOI : https://doi.org/10.4000/developpementdurable.8387

Ce document a été généré automatiquement le 21 septembre 2020.

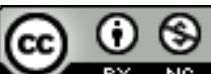

Développement Durable et Territoires est mis à disposition selon les termes de la licence Creative Commons Attribution - Pas d'Utilisation Commerciale 4.0 International. 


\title{
La coopération décentralisée face aux enjeux du développement durable
}

\author{
Introduction au numéro « Coopération décentralisée et développement \\ durable »
}

Decentralized cooperation and the challenges of sustainable development

\author{
Abdelkader Djeflat et Bruno Boidin
}

1 La coopération décentralisée a connu un nouvel élan et s'est développée à un rythme accéléré depuis la loi du 6 février 1992 en France. D'autres textes juridiques et réglementaires sont ensuite venus compléter le dispositif (notamment les lois de 1995, 1999 et 2000). Dans des domaines spécifiques comme celui de l'eau, d'autres dispositions, comme la loi dite Oudin-Santini ${ }^{1}$ de 2005 (qui autorise les collectivités locales, syndicats intercommunaux et agences de l'eau à consacrer jusqu'à $1 \% \mathrm{du}$ budget annexe de leurs services d'eau et d'assainissement au financement d'actions de coopération internationale dédiées au secteur de l'eau) se sont ajoutées.

2 La coopération décentralisée désigne toutes «les opérations de coopération mises en œuvre directement ou sous leur impulsion, ou avec leur soutien par des collectivités territoriales " (Petiteville, 1995). Elle est définie également par la loi du 6 février 1992 de la façon suivante: "il y a coopération décentralisée lorsqu'une (ou plusieurs) collectivité(s) locale(s) française(s) développe(nt) des relations avec une (ou plusieurs) collectivité(s) locale(s) étrangère(s) : il peut s'agir aussi bien de l'établissement de relations d'amitié ou de jumelage avec des collectivités territoriales étrangères, d'actions de promotion à l'étranger, d'aide au développement de collectivités dans certains pays, d'assistance technique, d'action humanitaire, de gestion commune de biens de services, mais aussi de coopération transfrontalière et de coopération interrégionale" (CNCD, 2000). La coopération décentralisée est venue combler un vide, compléter et corriger les dispositifs de coopération bilatérale et multilatérale qui existaient entre pays du Nord et pays du Sud et qui avaient commencé à s'essouffler et à montrer, dès les années 1980, de sérieuses limites et faiblesses. Au niveau des municipalités, les jumelages ont été érigés en instrument privilégiés de rapprochement 
entres les villes et ont constitué des plateformes de travail. Ceci correspondait à la fois aux besoins de développement urbain du Sud et à ceux de coopération du Nord.

3 L'avènement de la coopération décentralisée, perçue comme une étape supérieure par beaucoup d'analystes, constituait à la fois un moyen de résoudre les problèmes rencontrés par ces jumelages (en particulier la léthargie dans laquelle ils étaient entrés) et une façon de faire face aux besoins changeants en matière de coopération des villes du Nord autant que celles du Sud (Djeflat, 2007). Ces dernières restaient en effet entravées par leurs difficultés anciennes dans les domaines des voiries, de la gestion des déchets, de la distribution de l'eau ou encore des problèmes de circulation urbaine, pour ne citer que quelques exemples.

4 Le tissu associatif, traduisant le souhait des citoyens du Nord de s'impliquer dans des actions caritatives et de solidarité, est venu renforcer cet engouement pour les pays du Sud et a parfois intégré des espaces que les dispositifs de coopération mis en place pour les besoins des collectivités locales et territoriales n'étaient pas en mesure de couvrir. Ce tissu associatif a trouvé de plus en plus d'occasions de nouer des partenariats au Sud face, notamment, aux ouvertures démocratiques dans beaucoup de ces pays permettant l'aménagement progressif d'un espace public d'expression citoyenne avec différents niveaux d'ouverture. L'émergence d'un tissu urbain de plus en plus renforcé par l'exode rural et des institutions administratives locales plus ou moins calquées sur les schémas et les pratiques du Nord contribuaient à multiplier les opportunités de coopération. Parallèlement, la nécessité de prendre en charge des besoins multiples hors des centres urbains et auprès de populations rurales et villageoises, parfois en dehors de tout dispositif formel, devenait de plus en plus pressante.

5 Si ces mutations ont traduit une certaine volonté d'adapter le dispositif de la coopération décentralisée aux besoins des pays du Sud, les domaines de coopération ont, quant à eux, connu très peu d'évolution, insérés dans des dispositifs restés totalement ou partiellement figés aux plans règlementaire et institutionnel. Les domaines conventionnels cités plus haut (déchets, eau, voirie, etc.) sont restés les secteurs de prédilection de nombreuses opérations conduites dans les pays du Sud. Les problèmes abordés par les acteurs associatifs n'ont pas, eux non plus, connu d'évolutions notables au fil des années, demeurant limités à des projets conventionnels tels que le creusement de puits, la construction de salles de classe et de cases de santé ou la fourniture de médicaments et de manuels. Les soucis d'éducation au développement ont parfois fait évoluer ces pratiques, répondant à des besoins légitimes des pays du Nord et dont l'impact à long terme n'a pas matière à être fondamentalement questionné.

6 Cependant force est de reconnaître qu'un domaine a émergé depuis les accords de Rio (Sommet de la Terre, 1992), concomitant à la promulgation des lois sur la coopération décentralisée en France : celui du développement durable dont l'ascension fulgurante durant les vingt dernières années n'est plus à démontrer. Dans ce nouveau contexte, les réflexions et pratiques du développement vont être largement remises en question : les enjeux d'environnement, de durabilité des actions et programmes, de choix inter générationnels amènent les pays du Sud à entrer progressivement dans une nouvelle ère de questionnements et de remise en cause de certains schémas et approches du développement (Boidin, Djeflat, 2009).

7 La coopération décentralisée a ainsi investi des domaines liés au développement durable et cette thématique semble monter en puissance dans le cadre de l'appui au 
développement des pays du Sud (IEPF, 2007). Cette tendance reste toutefois relativement timide. Les questions d'adduction d'eau, d'assainissement, de gestion des déchets, notamment dans les centres urbains, font souvent l'objet de projets et de programmes de coopération entre des collectivités locales ou entre des ONG et des associations et leurs partenaires du Sud. Cependant, de nombreux projets n'intègrent pas ou peu les préoccupations de développement durable, soit par manque de sensibilisation des acteurs du Nord et du Sud, soit par faiblesse de moyens, soit encore sous la pression des besoins plus immédiats à satisfaire. L'approche "projet $»^{2}$ qui a longtemps dominé les politiques de coopération pour le développement, semble avoir focalisé l'attention sur le souci de répondre à des besoins de court terme exprimés par les populations du Sud, et parfois aux vœux des bailleurs de fonds (en particulier les collectivités du Nord, soucieuses de montrer des résultats concrets face à des élus parfois relativement réticents et peu convaincus de l'utilité de ces actions).

8 Pourtant, une coopération décentralisée n'a de sens que si elle s'inscrit dans une perspective de durabilité. Les projets doivent non seulement aider à résoudre les problèmes courants mais également permettre aux populations locales de les inscrire dans les perspectives de développement du village ou du territoire à long terme et dans le développement durable du pays. La coopération décentralisée ne devrait donc pas se traduire par des coûts sur l'environnement et sur la santé que les populations souvent démunies devront payer par la suite. Les actions de coopération décentralisée n'ont de sens, enfin, que si elles contribuent à développer des capabilities dans le sens d'Armatya Sen, ${ }^{3}$ à transférer des savoir faire dans la gestion des questions fortement liées à l'environnement et à assurer des revenus pérennes aux générations actuelles ainsi qu'aux générations futures.

9 Ce numéro spécial a pour objectif d'amener quelques éclairages et de souligner les enjeux majeurs dans ce domaine de croisement entre le champ de la coopération décentralisée et celui du développement durable. Initialement, le numéro était destiné à recueillir les travaux et réflexions tant des chercheurs que des praticiens ayant fortement capitalisé leurs expériences de terrain et acquis le recul nécessaire pour questionner le champ de la coopération décentralisée à partir d'une perspective de développement durable. Il est apparu qu'en plus de la difficulté liée à la nouveauté de cette thématique croisée, peu d'acteurs de terrain disposent de suffisamment de temps et de recul pour expliciter et codifier leurs connaissances et savoirs. Ceci illustre d'une manière indirecte le caractère à peine émergent d'une coopération décentralisée porteuse de valeurs du développement durable face à la non-durabilité des connaissances et à l'instabilité qui, parfois, caractérise le domaine de la coopération décentralisée.

10 Pourtant, le terrain de la coopération décentralisée, du fait de sa complexité croissante, interroge régulièrement les professionnels et les praticiens de la coopération et rend nécessaires l'investigation et l'analyse. Au plan de la recherche académique, les expériences de terrain accumulées sur de longues années constituent des gisements de données, de connaissances tacites encore peu codifiées que la rhétorique seule ne peut suffire à proprement investir. Plusieurs questions cruciales ont été encore peu abordées: quels schémas de coopération décentralisée pour un développement durable? Quels acteurs et institutions? Quel type de durabilité et quelles politiques publiques? Les travaux sur ces sujets amèneraient incontestablement un 
enrichissement tant sur le plan conceptuel que sur celui des approches méthodologiques.

11 Les contributions présentées dans ce numéro témoignent de cette interpénétration progressive entre coopération décentralisée et développement durable et posent des questions peu abordées dans le passé par le champ de la coopération décentralisée et celui du développement durable. Cette fertilisation croisée des deux domaines thématiques révèle un potentiel significatif d'enjeux collectifs et de terrains de recherche qu'il est utile de mettre en lumière pour les futurs travaux. ${ }^{4}$

C'est à partir de terrains spécifiques que la problématique du développement durable dans la coopération décentralisée est abordée dans ce numéro : le secteur de l'eau et sa "gouvernance ", les questions culturelles et enfin la problématique spatiale de l'espace euro-méditerranéen. Des pistes intéressantes et fécondes, susceptibles d'être approfondies et valorisées par des analyses théoriques et soutenues par l'investigation empirique, se dessinent: la gouvernance décentralisée des ressources naturelles et de l'environnement (Froger, 2010 ; Guesnier, 2010) appuyée sur les politiques et les projets environnementaux mis en œuvre dans les pays en développement ; la pénétration de la sphère de l'économie publique par l'économie solidaire ainsi que l'évaluation de la soutenabilité sociale (Makkaoui et Dubois, 2010); les perspectives de renforcement des compétences des autorités locales et de leur identité à travers l'ouverture sur l'extérieur et la confrontation des expériences (Soldo et Moustier, 2010); enfin, les pistes offertes par la Charte Européenne de la coopération qui met en avant des ingrédients fondamentaux de la durabilité que sont la participation des autorités locales dans la gestion concertée et durable des ressources naturelles, mais également la diversification des ressources financières, la préservation de l'identité nationale et des différentes formes de redevabilité (accountability) (Dusepulchre, 2010). Le couple développement durable coopération décentralisée se trouve ainsi investi de trois modes opératoires: une approche sectorielle mobilisant les domaines spécifiques comme celui de l'eau, une approche par les capacités des acteurs dans le sens de développer et renforcer les capabilities, enfin une approche institutionnelle dans sa mission internationale.

Premièrement, concernant l'approche sectorielle, la question de l'eau paraît présenter un terrain idéal pour le croisement des deux problématiques. C'est une question qui s'inscrit dans la continuité des engagements de Johannesburg (1992) et de Kyoto (1997), avec un développement des réflexions à l'échelle des régions en liaison avec l'ensemble des acteurs du territoire (Froger, 2010). L'évolution des besoins ponctuels des collectivités du Sud vers une véritable logique de développement durable local, conçu sous la forme de programmes multi-sectoriels, constitue une des mutations perceptibles à la fois du fait des thèmes choisis (développement territorial, tourisme responsable et solidaire, co-développement) que des modes opératoires (passage progressif de l'approche projet à l'approche programme) ou des logiques thématiques (gouvernance de proximité). Si cette tendance reste actuellement minoritaire dans l'ensemble des pays du Sud, la gouvernance décentralisée des ressources naturelles et de l'environnement reste cependant une problématique centrale dans les pays en développement avec des conflits de temporalité qui peuvent jouer à l'encontre de la durabilité. Le couple eau-développement durable nécessite une mobilisation générale dans la perspective d'un changement des modes de production et de consommation et de l'adoption d'un autre modèle de développement (Guesnier, 2010). La reconnaissance du droit des collectivités locales dans la gestion de l'eau reste un élément essentiel : ces 
dernières, proches des usagers, sont considérées comme les plus aptes à gérer l'approvisionnement, la collecte et l'entretien des réseaux d'eau. Cependant certains États ne sont pas prêts à décentraliser cette compétence.

Deuxièmement, l'approche par les capabilities pose, d'une part, la question première de la capacité des populations à satisfaire leurs besoins actuels et futurs, d'autre part, celle de la répartition des capacités entre différentes générations (Makkaoui et Dubois, 2010). Le renforcement des capabilities peut contribuer à la mise en œuvre d'un développement qui serait durable en termes sociaux et permettre de nouvelles formes de gestion participative. La coopération décentralisée a un rôle important à jouer dans ce contexte dans la mesure où, au delà des techniques et des savoir faire, elle doit procéder à un véritable transfert des valeurs, habituellement portées par l'économie solidaire, telles que la responsabilité, l'équité et la reconnaissance de la dignité de l'Autre. Cependant apparaissent quelques difficultés: la possibilité d'évaluer la soutenabilité sociale d'une telle expérience, l'adhésion de la population et sa mobilisation face à la réalisation des différents projets, et enfin le poids des bailleurs de fonds qui ont tendance à imposer leurs priorités et qui, parfois, obligent les acteurs locaux à réorienter leurs activités. Troisièmement, au plan institutionnel, des programmes internationaux spécifiques, bien que portés par des organisations multilatérales, peuvent avoir un impact non négligeable en termes de durabilité en encourageant la coopération décentralisée entre les autorités locales dans le cadre de divers programmes. C'est le cas du programme EUROMED Héritage dont la mission est d'aider les pays méditerranéens dans leurs initiatives de promotion et de préservation de leur patrimoine, permettant aussi d'éduquer la société civile à la sauvegarde du patrimoine (Soldo et Moustier, 2010). La coopération culturelle est au centre des préoccupations. La durabilité est assurée, dans ce contexte, par un certain nombre d'éléments : effets d'apprentissage et transferts de connaissance, liens de coopération pérenne à partir d'initiatives propres aux pouvoirs locaux, renforcement de leurs compétences et de leur identité à travers l'ouverture sur l'extérieur ainsi que la confrontation des expériences. Les questions centrales sont ici relatives à la capacité de la coopération culturelle à opérer des ruptures vis-vis des pratiques actuelles et à être le fruit d'une véritable concertation.

Enfin, toujours au plan institutionnel, la Charte européenne de la coopération insiste sur le renforcement de la "participation des autorités locales dans la gestion concertée et durable des ressources naturelles, dans le respect des politiques nationales». Elle pose un certain nombre de principes d'action dont les effets sur la durabilité sont incontestables (Dusepulchre, 2010, p. 1) la diversification des ressources, de telle sorte qu'elles soient constituées par des rentrées fiscales propres, des fonds extérieurs (aide extérieure et marchés financiers), des transferts nationaux et une politique financière transparente. 2) La prise en compte des réalités et aspirations des autorités locales des pays en développement et l'identification des stratégies qui répondraient le mieux à leurs préoccupations. 3) L'adoption des principes de subsidiarité et des différentes formes d'accountability comme moyens de renforcer leur gouvernance locale (ascendante, horizontale et descendante).

Deux écueils doivent néanmoins être évités dans ce contexte: le premier est la tendance des États européens à exporter les modèles d'autonomie locale dans des Etats tiers qui n'ont eu ni l'occasion de participer à leur élaboration, ni l'opportunité d'y introduire toutes les nuances utiles à la préservation de leur identité nationale. Le 
deuxième est l'existence d'idéologies et de schémas pré-établis de gouvernance qui reposent peu sur des bases empiriques éprouvées ou une connaissance suffisante des mécanismes à l'œuvre dans les pays en développement. À titre d'exemple, le FMI et la Banque mondiale ont établi et promu un programme de "gouvernance " pour les pays en développement depuis la fin des années 1990. Cet agenda s'inspire en partie de la « nouvelle économie institutionnelle (Williamson, 2000) et tente de proposer un cadre théorique unifié regroupant des termes aussi variés que la "bonne gouvernance », les incitations, la démocratie, la participation des acteurs, la décentralisation. Or ce modèle pré-établi comporte des failles théoriques et constitue un agenda clé en main souvent déconnecté des spécificités historiques, institutionnelles et économiques des pays en développement.

\section{BIBLIOGRAPHIE}

Ballet J., Dubois J.L., Mahieu F.R., 2004, « À la recherche du développement socialement durable : concepts fondamentaux et principes de base », Développement durable et territoires, http:// developpementdurable.revues.org/document1165.html

Boidin A., Djeflat A., 2009, « Spécificités et perspectives du développement durable dans les pays en développement », Mondes en Développement, n¹48, 4/ 2009

CNCD (Centre National de la Coopération Décentralisée), 2000, Guide de la coopération décentralisée, Paris, La Documentation française.

Djeflat A., 2007, « La coopération décentralisée face aux besoins changeants des pays du Sud », in B. Gallet, A. Bekkouche et Villard Y. (eds.), La coopération décentralisée change-t-elle de sens?, Collection référence, Citées Unies France, 354 pages

Djeflat A., 2008, « La Coopération décentralisée face aux chocs exogènes », Cahiers de la Coopération Décentralisée, Cités Unies France, Vol. I n¹, pp.

Dusepulchre G., 2010, « La charte européenne de la coopération en matière d'appui à la gouvernance locale : outil et questionnements pour les autorités territoriales ", Développement Durable et Territoires, vol. $1, \mathrm{n}^{\circ} 1$

IEPF, 2007, Coopération décentralisée et développement durable, numéro spécial de la Revue Liaison Energie-Francophonie, 77(4), 101 p.

Froger G., 2010, « Les coopérations au développement durable en question dans les pays du Sud », Développement Durable et Territoires, vol. $1, \mathrm{n}^{\circ} 1$

Guesnier B., 2010, «L'eau et le développement durable : un couple en rupture sans gouvernance sociétale et coopération décentralisée ", Développement Durable et Territoires, vol. 1, nº

Maders H.-P., 2003, Manager une équipe projet, troisième édition, Eyrolles, Paris.

Makkaoui-Mathieu R., Dubois J.-L., 2010, « Nouvelles formes de gouvernance dans le domaine de l'eau. Apports et limites de la coopération décentralisée dans les pays en développement », Développement Durable et Territoires, vol. $1, \mathrm{n}^{\circ} 1$ 
Petiteville F. 1995, « La coopération décentralisée : les collectivités territoriales dans la coopération », l'Harmattan, Paris.

Sen A., 1981, Poverty and Famines: An Essay on Entitlement and Deprivation, New York: Oxford University Press.

Sen A., 1985, Commodities and capabilities, Amsterdam, North-Holland, 93 p.

Sen A., 2000, Un nouveau modèle économique, Paris, Odile Jacob, 479 p.

Soldo E., Moustier, E., 2010, « La coopération décentralisée : un élan pour le développement culturel durable dans l'espace euro-méditerranéen », Développement Durable et Territoires, vol. 1, $\mathrm{n}^{\circ} 1$

Williamson O., 2000, “The new institutional economics. Taking Stock, Looking Ahead”, Journal of Economic Literature, vol. 38, September, p. 595-613.

\section{NOTES}

1. Promulguée le 27 janvier 2005.

2. Elle consiste à gérer les actions de coopération sur la base d'un projet c'est-à-dire sous forme d'un « ensemble finalisé d'activités et d'actions entreprises dans le but de répondre à un besoin défini, dans des délais fixés et dans la limite de l'enveloppe budgétaire allouée » (Maders, 2003).

3. Les capabilities définissent l'ensemble des actes que tout individu devrait être capable d'accomplir (Sen, 1985, 2000). Ballet, Dubois et Mahieu (2004) considèrent que la faiblesse des capabilities dans certains domaines (par exemple la santé) empêche la conversion des capabilities en fonctionnements dans d'autres domaines (économique, environnemental, social).

4. En France, ceci constitue également pour le champ de la coopération décentralisée une opportunité à saisir du fait que les mutations récentes des diplômes rendent les lignes entre formation professionnelle et formation à la recherche beaucoup moins nettes que par le passé. Les nouvelles maquettes de formation habilitées pour 2010-2014 ne font plus de différence entre Master professionnels et Masters recherche. Il n'existe dorénavant plus qu'un seul Master qui permet de choisir soit une intégration au marché du travail soit une poursuite en thèse en vue de l'obtention d'un doctorat. 